\title{
Modelling of hydraulic governor-turbine for control stabilisation
}

\author{
Yin Chin Choo $^{1} \quad$ Kashem M. Muttaqi ${ }^{2}$ \\ M. Negnevitsky ${ }^{3}$
}

(Received 27 July 2007; revised 22 July 2008)

\begin{abstract}
Power system performance is affected by dynamic characteristics of hydraulic governor-turbines during and following any disturbance, such as occurrence of a fault, loss of a transmission line or a rapid change of load. Accurate modelling of hydraulic governor-turbines is essential to characterise and diagnose the system response during an emergency. In this article, both detailed and simplified hydraulic systems governed by proportional-integral-derivative and proportionalintegral controllers are modelled. This article examines their transient responses to disturbances through simulation in Matlab/Simulink. The article also investigates the dynamic performance of an isolated hydraulic system through evaluating stability margins, eigenvalues, root loci and frequency deviation time responses of the system. The results obtained provide an insight into the stability of the power system governed by different governor settings.
\end{abstract}

See http://anziamj.austms.org.au/ojs/index.php/ANZIAMJ/article/view/333 for this article, (c) Austral. Mathematical Soc. 2008. Published August 13, 2008. ISSN $1446-8735$ 


\section{Contents}

1 Introduction

C682

2 Mathematical representations of hydraulic system

C683

2.1 Hydraulic turbine model . . . . . . . . . . . . . . . C684

2.1.1 Realistic nonlinear turbine-penstock model . . . . . C684

2.1.2 Linearised turbine-penstock models . . . . . . . . . C687

2.2 Hydraulic governor model . . . . . . . . . . . . . . C688

2.3 Combined electrical system . . . . . . . . . . . . C690

3 Simulation results

C691

4 Conclusions

C696

References

C696

\section{Introduction}

Since 1973, the demand on modelling requirements for power system components has become more onerous due to the increasing complexity of power system networks [4]. Power system components such as the governor, turbine and generator need to be represented by a detailed model. Classical representations of a hydraulic system, which assume an ideal lossless turbine, are limited to small perturbations around an initial operating point. They depict the actual characteristics at only very low frequencies. For large signal stability studies, the classical model does not capture the dynamic behaviour accurately and leads to erroneous responses. Moreover, conventional governor tuning is performed based on the classical model, hence the optimal and stable governor settings obtained by the classical model differ substantially from the settings obtained from comprehensive models. It is thus vital to represent the hydraulic model in detail in order to determine 


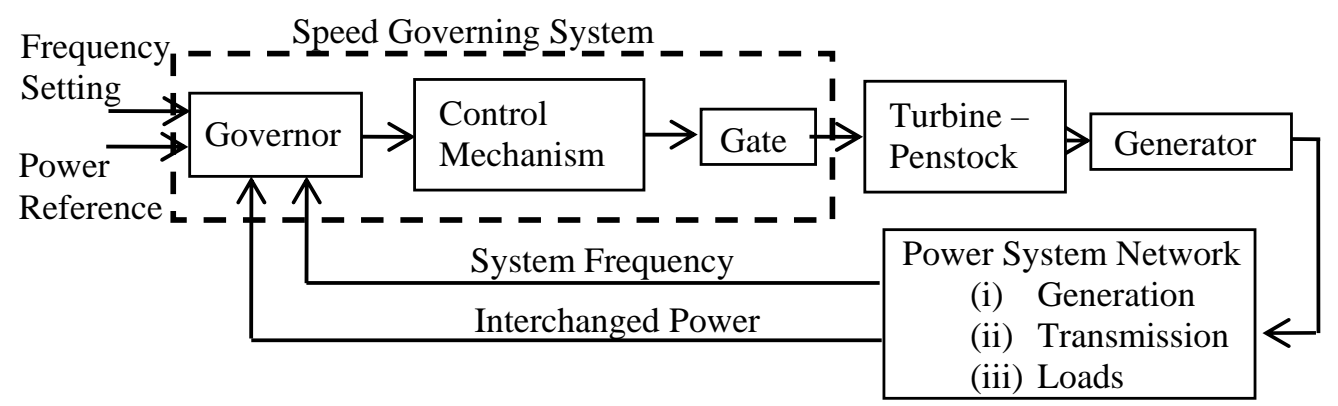

FiguRE 1: Functional block diagram of hydraulic governor-turbine system interconnected with a power system network.

the practical dynamic responses as accurately as possible for a wide variety of system studies.

This article presents a detailed mathematical representation of the hydraulic turbine-penstock. The dynamic performance of the hydraulic system is studied using time domain and frequency domain methods.

\section{Mathematical representations of hydraulic system}

In this section, a mathematical representation of a hydraulic system, including both turbine-penstock and the governing system, is introduced. Figure 1 shows a block diagram of the hydraulic governor-turbine system connected to a power system network. The primary source for the electrical power provided by utilities is the kinetic energy of water which is converted into mechanical energy by the prime movers. The electrical energy to be supplied to the end users is then transformed from mechanical energy by the synchronous generators. The speed governing system adjusts the generator speed based on the input signals of the deviations of both system frequency 
and interchanged power with respect to the reference settings. This is to ensure that the generator operates at or near nominal speed at all times.

\subsection{Hydraulic turbine model}

The dynamic performance of a hydraulic system is affected by the turbinepenstock characteristics which are determined by water inertia, water compressibility, and pipe wall elasticity in the penstock. The effects of each component need to be modelled carefully to study their impact on the system dynamic performance. For example, water inertia causes changes in turbine flow to lag behind changes in turbine gate opening, and the travelling waves of pressure and flow involving a compressible fluid in an elastic pipe lead to the water hammer phenomenon.

\subsubsection{Realistic nonlinear turbine-penstock model}

An IEEE working group [4] and Kundur [5] discussed the model for a detailed representation of hydraulic dynamics in the penstock. The hydraulic unit characteristic of a single penstock is [5]

$$
\begin{aligned}
(d q / d t) & =\left(h_{0}-h-h_{l}\right) a_{\text {gravity }} A / L, \\
\bar{q} & =\bar{G} \sqrt{\bar{h}} .
\end{aligned}
$$

The terms for the physical design of the plant describe the water starting time constant for rated conditions when equation (1) is normalised using the per unit system [2]

$$
d \bar{q} / d t=\left(1-\bar{h}-\bar{h}_{l}\right) / T_{\text {w_rated }},
$$

where $T_{\text {w_rated }}=L q_{\text {base }} /\left(a_{\text {gravity }} A h_{\text {base }}\right)$. 


\section{TABle 1: Nomenclature}

$q=$ turbine flow

$G=$ ideal gate opening based on the change from the no load to full load being equal to 1 per unit [\% or pu] $g=$ movement of wicket gate or the amount of gate opening/closing [\% stant [sec]

or $\mathrm{pu}]$

$h=$ hydraulic head at gate

$h_{l}=$ head losses due to friction in the conduit

$h_{0}=$ initial steady-state head

$P_{m}=$ mechanical power

$P_{e}=$ electrical power

$a_{\text {gravity }}=$ gravity acceleration

$L=$ conduit length

$A=$ penstock cross section area

$T_{m}=$ mechanical starting time [sec] $T_{w}=$ Water starting time constant [sec]

$T_{\text {w_rated }}=$ water starting time constant

$t=$ time [sec]

$\omega_{\text {ref }}=$ reference/nominal speed

$\omega=$ shaft speed

$D=$ Self-regulation of load

$\sigma=$ permanent speed droop $=0.04$

$K_{p}=$ proportional gain $=3.0$
$K_{i}=$ integral gain $=0.7$

$K_{d}=$ derivative gain $=0.5$

$T_{A}=$ pilot servomotor time con-

$T_{C}=$ gate servo gain [sec]

$T_{D}=$ gate servomotor time constant [sec]

$\bar{q}_{\text {noload }}=$ per unit no load water flow

$q_{\text {base }}=$ base value of water flow

$h_{\text {base }}=$ base value of hydraulic head

$A_{t}=$ turbine gain

$\bar{g}_{\text {FullLoad }}=$ per-unit full-load gate opening

$\bar{g}_{\text {NoLoad }}=$ per-unit no-load gate opening

- denotes normalised value [pu]

^denotes Laplace notation

Laplace Transform variable is denoted ' $s$ '

$$
\begin{aligned}
& a_{11}=\partial q / \partial h \\
& a_{12}=\partial q / \partial \omega \\
& a_{13}=\partial q / \partial g \\
& a_{21}=\partial P_{m} / \partial h \\
& a_{22}=\partial P_{m} / \partial \omega \\
& a_{23}=\partial P_{m} / \partial g
\end{aligned}
$$




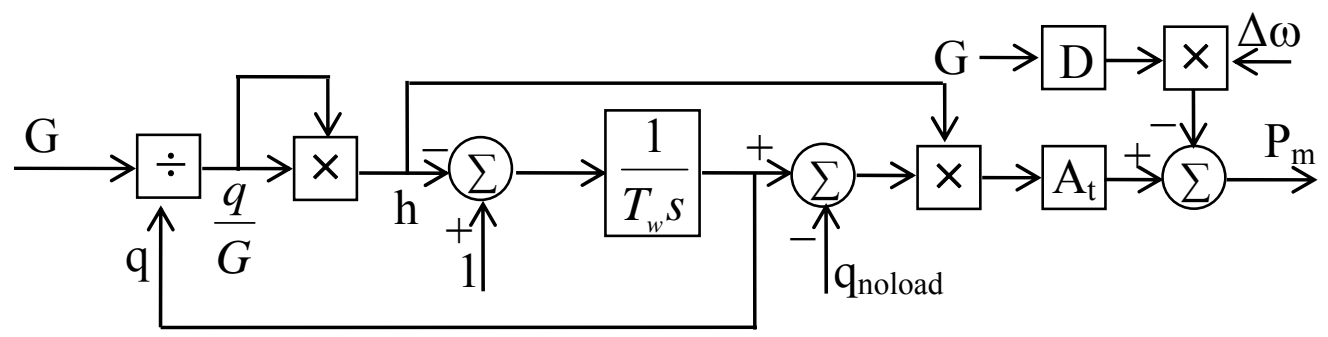

FiguRe 2: Hydraulic turbine block diagram assuming an inelastic water column.

The turbine representation is based on steady state measurements related to output power and water flow [2]

$$
\bar{P}_{m}=A_{t} \bar{h}\left(\bar{q}-\bar{q}_{\text {noload }}\right),
$$

where turbine gain $A_{t}$ is a proportionality factor and is assumed to be constant, that is $A_{t}=1 /\left(\bar{g}_{\text {FullLoad }}-\bar{g}_{\text {NoLoad }}\right)$.

In practice, it is common for governor-turbine manufacturers to indicate the speed ring position by arranging the gate position sensing device to give readings as a fraction of the gate stroke from the fully closed to the fully opened setting. In reality, the gates will not be fully closed at no load and they may not be completely opened at rated load. Hence, Undrill and Woodward [7] assumed that the turbine torque at rated speed and rated head is linearly related to the gate position sensor reading.

The combination of equations (2), (3) and (4) yields the general dynamic characteristics of a hydraulic turbine with a penstock, unrestricted head and tail race as shown in the block diagram in Figure 2. A supplementary term, GD $\Delta \omega$, is subtracted from the output of the turbine model to represent speed deviation damping due to gate opening [4]. The speed deviation $\Delta \omega$ is the deviation of the actual turbine-generator speed from the nominal speed. 


\subsubsection{Linearised turbine-penstock models}

The dynamic characteristics of the power system components are normally represented mathematically by differential equations. The technique of linear system analysis is employed to investigate the dynamic behaviour of the power system assuming small signals. The Laplace transform is used herein to solve the linear differential equations; this method replaces the more complicated differential equations with relatively easily solved algebraic equations.

For a small variation about an equilibrium condition, the turbine is represented by the linearised equations, normalised based on initial steady state values $[4,5,6]$

$$
\begin{aligned}
\Delta \bar{q} & =a_{11} \Delta \bar{h}+a_{12} \Delta \bar{\omega}+a_{13} \Delta \bar{g}, \\
\Delta \bar{P}_{m} & =a_{21} \Delta \bar{h}+a_{22} \Delta \bar{\omega}+a_{23} \Delta \bar{g} .
\end{aligned}
$$

Both the water flow and the mechanical power of the turbine are functions of head $h$, machine speed $\omega$ and gate opening $g$. The partial derivatives represented by coefficients $a_{1 i}$ and $a_{2 i}(i=1,2,3)$ is obtained from Thorne and Hill [6] for three different operating points.

In an interconnected power system, the hydraulic units are synchronised to the system network. As a result, speed variations $\Delta \omega$ are fairly small and usually neglected.

Consequently, the mechanical power output of the turbine is obtained, with the inclusion of water inertia effect [6]

$$
\Delta \hat{\bar{P}}_{m} / \Delta \hat{\bar{g}}=a_{23}\left\{\left[\left(a_{23} a_{11}-a_{21} a_{13}\right) / a_{23}\right] \hat{T}_{w} s+1\right\} /\left(a_{11} \hat{T}_{w} s+1\right) .
$$

Equation (7) is a conventional expression for a classical non-ideal hydraulic turbine. The superhat ^ denotes the notation of the Laplace transform parameters. Typical values of the coefficients for an ideal lossless turbine are 
$a_{11}=0.5, a_{13}=1.0, a_{21}=1.5$ and $a_{23}=1.0$. The ideal lossless turbine used in system stability studies is [6]

$$
\Delta \hat{\bar{P}}_{m} / \Delta \hat{\bar{g}}=\left(-\hat{T}_{w} s+1\right) /\left(0.5 \hat{T}_{w} s+1\right) .
$$

The water starting time constant used for the linearised turbine-penstock model corresponds to the hydro unit's operating condition, that is, $T_{w}$ is calculated with the current operating flow and head. For the nonlinear model described in Subsection 2.1.1, $T_{\text {w-rated }}$ is obtained at rated conditions using rated head and rated flow as the bases. The application of the nonlinear model for simulation analysis will be beneficial as it allows one data base to denote the dynamic models for the system independent of dispatch. This is true if there are no common flow paths for more than one unit at the plant; for example, a shared penstock. This is not the case for a linearised model where different values of $T_{w}$ are required as the dispatch of the hydro units is varied in the initial state load flows [2].

\subsection{Hydraulic governor model}

The fundamental function of a governor is to control the speed and/or load through the feedback signal of the speed error and/or power variation to control the gate position, which regulates the water flow through the penstock [5]. This is to ensure the active power balance in the system as well as to maintain grid frequency within nominal value under electrical load variations.

An electrohydraulic governor provided with proportional integral derivative (PID) controllers is shown in Figure 3. The speed deviation is processed by the PID terms into a command signal to a series of hydraulic valves and servos to produce a change in throttle valve or gate position of the prime mover. The time constants $T_{A}, T_{C}$ and $T_{D}$ of these hydraulic actuators are established by the pressure/flow characteristics of the gate and its servos. 


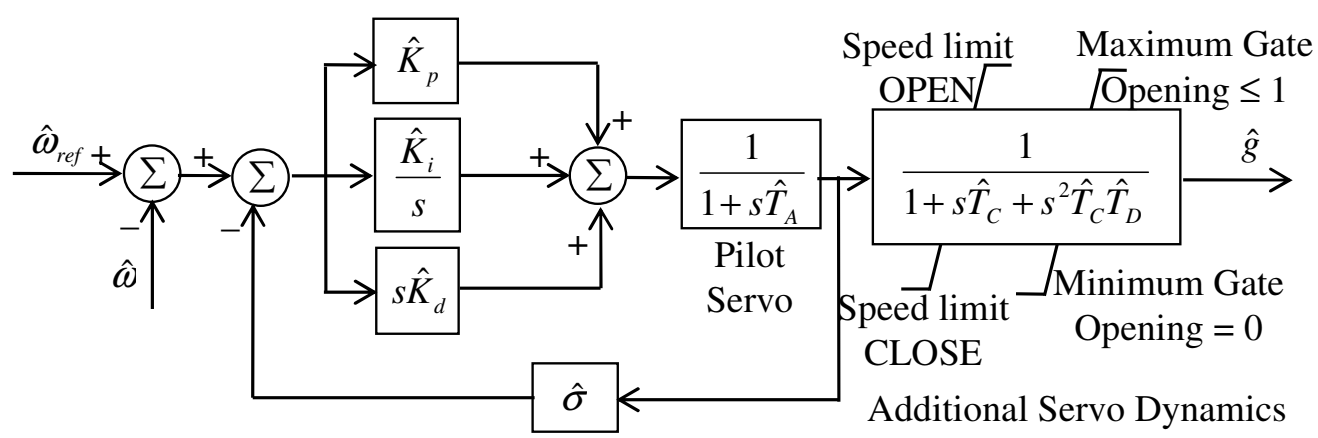

FIGURE 3: PID governor system.

However, the servomotor time constants have been neglected in deriving the governor response as their effects are substantial only if their poles occur before or near crossover frequency [4]. For security reasons, the speed of the gate stroke is restricted; it is represented by a simple rate-limit, which is sufficient for analysis. The gate opening is limited before it reaches its physical limits. Speed sensing, permanent droop, other measuring and computing operations are performed electrically. The electrical components provide better flexibility and enhanced execution with regard to dead bands and time lags [5]. The proportional term generates an instantaneous response to a speed error input, and it has a significant impact on the system stability. The integral term performs together with the proportional term to determine the stability. Its ability to reduce the speed error to a zero value is known as 'reset'. The derivative term allows the expansion of the stability limits with larger values of proportional and integral gains whilst retaining the system stability [3].

The occurrence of a power system fault, which is reflected in the frequency deviation $\left(\omega_{\text {ref }}-\omega\right)$, results in compensation operated by the PID governor. The correction executed depends on the droop, the dynamic settings and the characteristics of the PID governor. The permanent droop determines the speed regulation under steady state operating conditions. It is described as 
the percentage or per unit speed drop needed to drive the gate from minimum to maximum opening without varying the speed reference [4]. Given the turbine characteristics, PID gains act to give stable gate position control and follow command signals in increasing or decreasing power generation.

The PID governor is [5]

$$
\hat{g} /\left(\hat{\omega}_{\text {ref }}-\hat{\omega}\right)=\left(s^{2} \hat{K}_{d}+s \hat{K}_{p}+\hat{K}_{i}\right) /\left[s^{2} \hat{\sigma} \hat{K}_{d}+s\left(\hat{\sigma} \hat{K}_{p}+1\right)+\hat{\sigma} \hat{K}_{i}\right] .
$$

With derivative gain excluded, it yields the PI governor

$$
\hat{g} /\left(\hat{\omega}_{\text {ref }}-\hat{\omega}\right)=\left(s \hat{K}_{p}+\hat{K}_{i}\right) /\left[s\left(\hat{\sigma} \hat{K}_{p}+1\right)+\hat{\sigma} \hat{K}_{i}\right] .
$$

Small signal analysis is considered in the studies. The perturbations applied to the system are not significant such that neither the limits of the gate position nor the maximum gate opening/closing rates are reached. Thus, the windup effects have not been accounted for in the equations.

\subsection{Combined electrical system}

The differential equation of speed $\omega$ is derived and solved utilising water acceleration of the turbine and governor response described in Subsections 2.1 and 2.2 respectively, together with the swing equation depicting the machine acceleration $d \omega / d t=\left(P_{m}-P_{e}-D \omega\right) / T_{m}[5]$. This equation determines the network system stability.

The differential equation is transformed into Laplace space as

$$
\begin{array}{r}
0.5 \frac{\hat{\sigma} \hat{K}_{d}}{\hat{T}_{w}} s^{4} \hat{\omega}+\left(\frac{\hat{\sigma} \hat{K}_{d}}{\hat{T}_{w}}+0.5 \frac{\hat{K}_{d} \hat{D} \hat{\sigma}}{\hat{T}_{m}}+0.5+0.5 \hat{K}_{p} \hat{\sigma}-\frac{\hat{K}_{d}}{\hat{T}_{m}}\right) s^{3} \hat{\omega} \\
\left(\frac{\hat{D} \hat{\sigma} \hat{K}_{d}}{\hat{T}_{m}}+1+\hat{K}_{p} \hat{\sigma}+0.5 \frac{\hat{D} \hat{T}_{w}}{\hat{T}_{m}}+0.5 \frac{\hat{K}_{p} \hat{T}_{w} \hat{D} \hat{\sigma}}{\hat{T}_{m}}+0.5 \frac{\hat{K}_{p} \hat{K}_{i} \hat{\sigma} \hat{T}_{m}}{\hat{T}_{w}}+\right.
\end{array}
$$




$$
\begin{aligned}
& \left.+\frac{\hat{K}_{d}}{\hat{T}_{m}}-\frac{\hat{K}_{p} \hat{T}_{w}}{\hat{T}_{m}}\right) s^{2} \hat{\omega}+\left(\frac{\hat{D} \hat{T}_{w}}{\hat{T}_{m}}+\frac{\hat{K}_{p} \hat{T}_{w} \hat{D} \hat{\sigma}}{\hat{T}_{m}}+\frac{\hat{K}_{p} \hat{K}_{i} \hat{\sigma} \hat{T}_{m}}{\hat{T}_{w}}+0.5 \hat{K}_{p} \hat{K}_{i} \hat{D} \hat{\sigma}\right. \\
& \left.+\frac{\hat{K}_{p} \hat{T}_{w}}{\hat{T}_{m}}-\hat{K}_{p} \hat{K}_{i}\right) s \hat{\omega}+\left(\hat{K}_{p} \hat{K}_{i}+\hat{K}_{p} \hat{K}_{i} \hat{D} \hat{\sigma}\right) \hat{\omega}=0 .
\end{aligned}
$$

This has the fourth order polynomial form $a_{0} s^{4}+a_{1} s^{3}+a_{2} s^{2}+a_{3} s^{1}+a_{4}=0$, and it yields the stability limit if $\left(a_{1} a_{2}-a_{0} a_{3}\right) a_{3}-a_{12} a_{4}=0$ by the RouthHurwitz criterion. In control theory, the system is stable when all roots of the characteristic equation lie in the left half of the $s$-plane. Moreover, to ensure stability, all the coefficients of the characteristic equation need to have the same sign and must be nonzero [1].

\section{Simulation results}

Isolated operation of the hydraulic system is used for the case studies. A hydraulic turbine with water starting time, $T_{w}=2 \mathrm{sec}$, and mechanical starting time, $T_{m}=8 \mathrm{sec}$, is considered. A purely resistive electrical load with immediate voltage regulation is assumed with self-regulation $D=0$. This is a worse case scenario as the power is independent of the speed and the inverse relationship between torque and speed makes it adverse for system stability. MATLAB/Simulink is used as a software tool for simulation studies.

The unit impulse involved in the hydraulic turbine-governor transfer functions has the value of infinity at $t=0$ and zero for all other times. However, this does not appear in physical systems. In reality there is a very high finite value for a short period of time. Hydro turbines typically have initial inverse response characteristics of power to gate changes. They require provision of transient droop features in the speed controls for stable control operation; that is, the governor should exhibit high regulation (low gain) for rapid changes in the frequency and vice versa [4]. Thus the impulse functions involved in the turbine-governor equations are the natural response of 


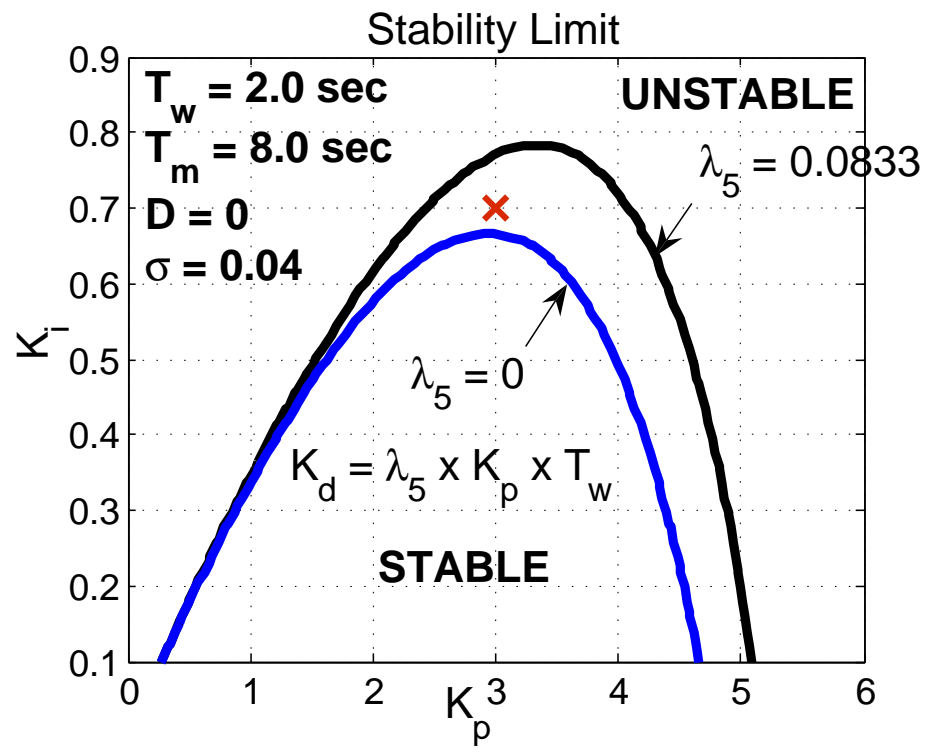

FiguRE 4: Stability limit of the hydraulic governor system.

the hydraulic system and the existing system transients. Practical systems normally operate under steady state condition with an operating point. If any fault occurs, the system will be perturbed from this operating point.

From a simulation perspective, the system will be initialised to avoid any unexpected response or instability due to the impulse functions as well as to restore the system operating conditions to a steady state level. These initial transients last for a short time only and are usually ignored as they do not represent the true behaviour of the system. Also, any inherently unstable responses and transients in the dynamic systems will be damped out by the amortisseurs winding of the machine rotors and the interconnected system. The huge inertia of the machine aids in the damping process as well.

Figure 4 shows the stability limit curves of the hydraulic system where a classical turbine model is utilised. For governor settings bounded within the 


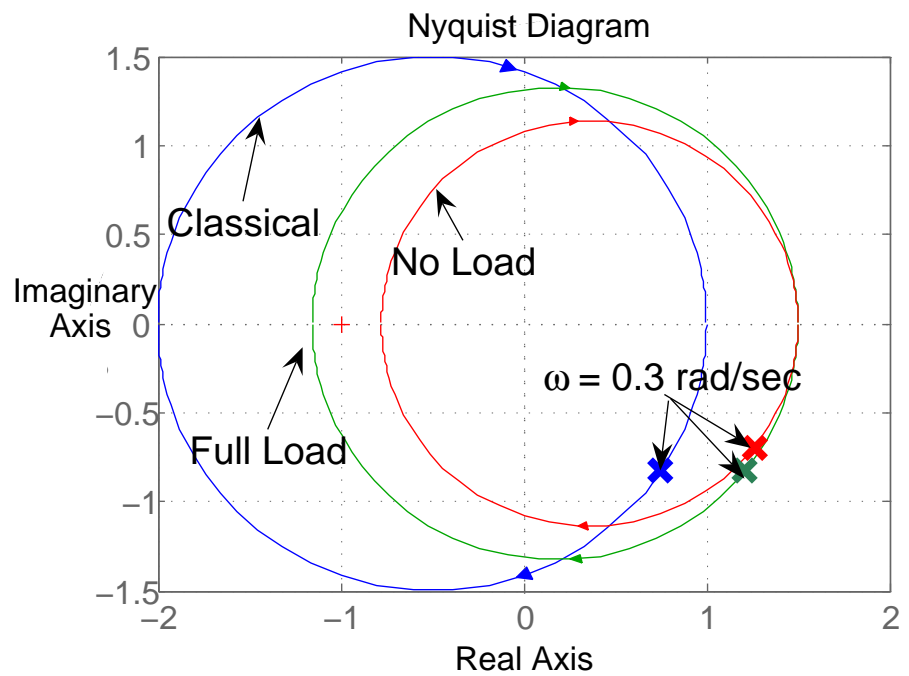

FigURE 5: Frequency response loci of hydraulic turbine-penstock models.

stability limit curve, a stable system will result. Governor settings on the stability limit result in an undamped oscillatory response. The inclusion of the derivative gain $K_{d}$ extends the stability boundary. With $K_{d}=0.5$, the governor settings with $K_{p}=3.0$ and $K_{i}=0.7$ result in a stable response. Instability results when the derivative gain is neglected.

Figure 5 shows the frequency response loci of different linearised hydraulic turbine-penstock models. The response for the classical model differs significantly from the linearised models operating at full load and no load for all frequency ranges. The linearised models agree well at fairly low frequencies. At a frequency of $0.3 \mathrm{rad} / \mathrm{sec}$, the linearised models operating at no load and full load share similar characteristics. However, their characteristics differ substantially with that of the classical model as the water column effect is not considered in the classical model.

Figure 6 shows the eigenvalues for the closed loop hydraulic governorturbine systems. The oscillatory mode represented by a pair of complex 
Eigenvalues Trace

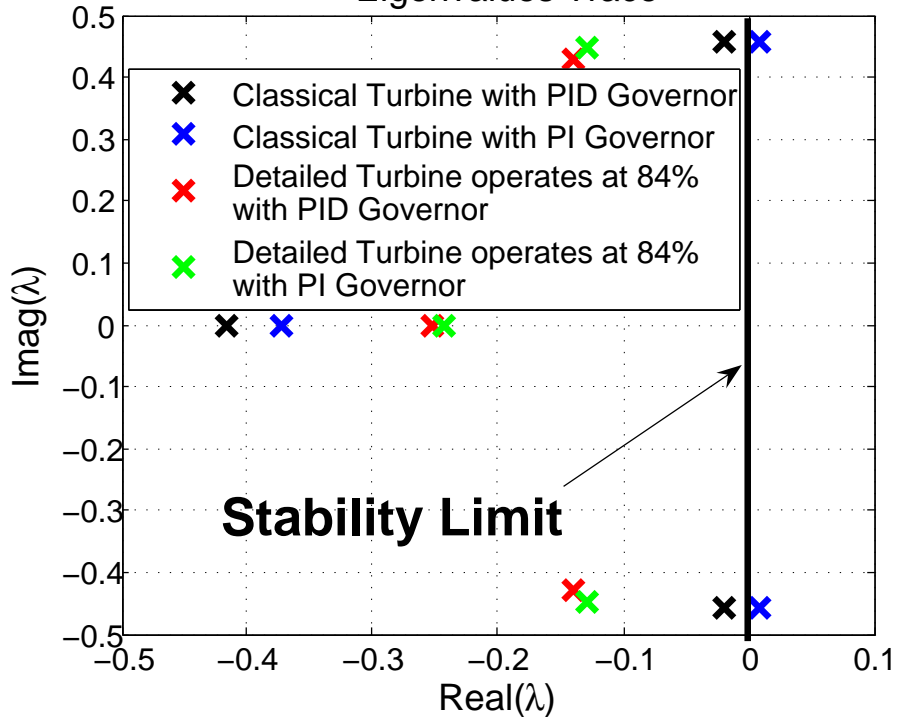

FiguRE 6: Eigenvalues trace for hydraulic system.

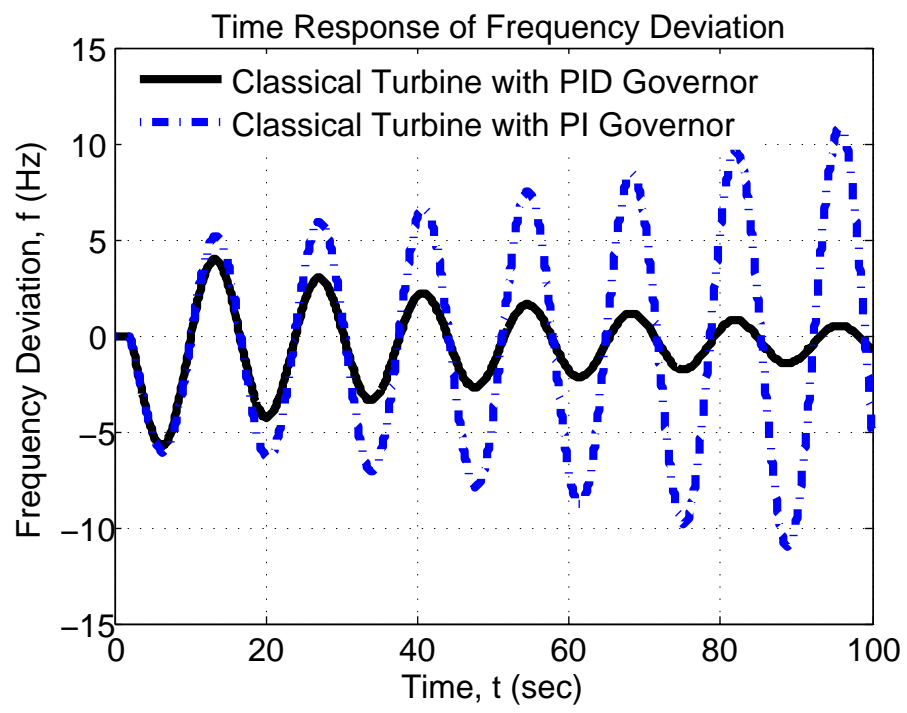

Figure 7: Frequency deviation time responses. 


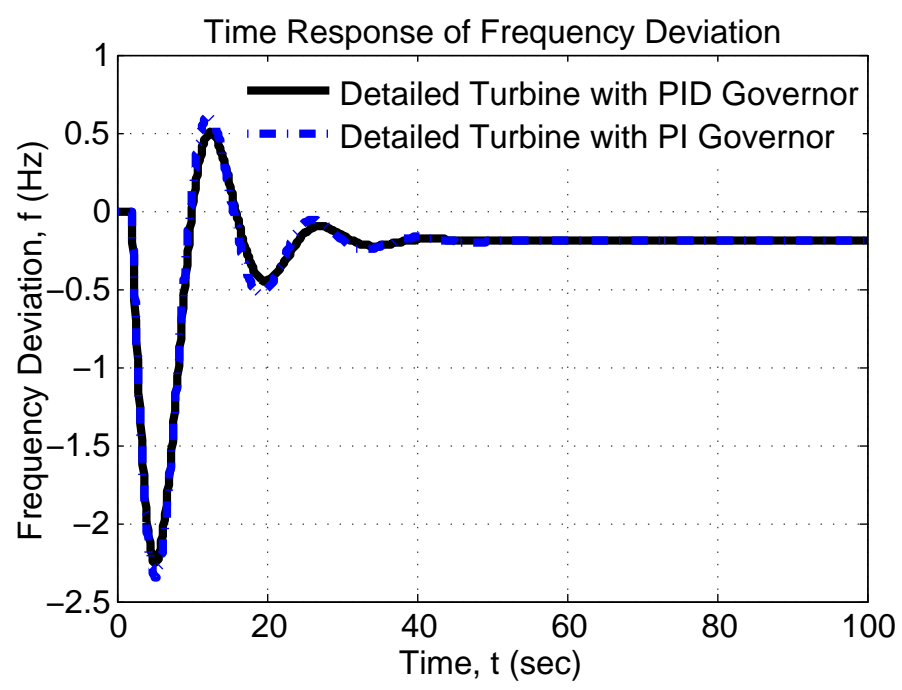

Figure 8: Frequency deviation time responses.

conjugate eigenvalues is of primary interest, as the real part of the eigenvalues is the amount of the associated damping, whilst the imaginary part measures the frequency of oscillations. The classical hydraulic system with $K_{d}$ neglected is unstable as a pair of the eigenvalues crosses over the stability limit. However, with the consideration of a detailed turbine model, both systems (with or without $K_{d}$ ) become stable. These results are verified by the frequency deviation time responses as shown in Figures 7 and 8, when a small amount of load is applied at time, $t=2 \mathrm{sec}$. An oscillatory but stable response for the classical turbine governed by the PID controller is observed in Figure 7. With a detailed turbine model, the responses reach steady state faster with less oscillation, and the deviations resulting are smaller as well. This comprehensive model incorporates the general dynamic characteristics of a hydraulic turbine with due consideration of the penstock, unrestricted head and tail race effects, and is suitable for most power system analysis. 


\section{Conclusions}

The time domain analyses of the governing system subject to load disturbances are verified by frequency response methods as well as with the stability limit curves of the hydraulic system. The detailed modelling of power system components is vital to capture essential system dynamic behaviour. The PID and PI governors perform better when applied to a more realistic model. Furthermore, the inclusion of the derivative action in the governor aids in extending the system stability limit.

Acknowledgements This research was supported by the Australian Research Council under ARC Discovery Grant K0014733, "Optimising Control of Hydroelectric Turbines Subject to Basslink Instability".

\section{References}

[1] Richard C. Dorf and Robert H. Bishop. Modern Control Systems. Pearson Educational, Inc, 2005. C691

[2] L. N. Hannett, J. W. Feltes, and B. Fardanesh. Field tests to validate hydro turbine-governor model structure and parameters. IEEE Transactions on Power Systems, 9(4):1744-1751, November 1994. doi:10.1109/59.331426. C684, C686, C688

[3] IEEE. IEEE guide for the application of turbine governing systems for hydroelectric generating units. IEEE Std 1207-2004, pages 1-121, 2004. C689

[4] IEEE. Hydraulic turbine and turbine control models for system dynamic studies. IEEE Transactions on Power Systems, 7(1):167-179, Feb 1992. doi:10.1109/59.141700. C682, C684, C686, C687, C689, C690, C691 
[5] P. Kundur. Power System Stability and Control. McGraw-Hill, 1994. C684, C687, C688, C689, C690

[6] D. H. Thorne and E. F. Hill. Field testing and simulation of hydraulic turbine governor performance. IEEE Transactions on Power Apparatus and Systems, PAS-93(4):1183-1191, July 1974. doi:10.1109/TPAS.1974.294066. C687, C688

[7] J. M. Undrill and J. L. Woodward. Nonlinear hydro governing model and improved calculation for determining temporary droop. IEEE Transactions on Power Apparatus and Systems, PAS-86(4):443-453, April 1967. doi:10.1109/TPAS.1967.291853. C686 


\section{Author addresses}

1. Yin Chin Choo, Integral Energy Power Quality and Reliability Centre, School of Electrical, Computer and Telecommunications Engineering, University of Wollongong, Australia. mailto:ycc260@uow.edu.au

2. Kashem M. Muttaqi, Integral Energy Power Quality and Reliability Centre, School of Electrical, Computer and Telecommunications Engineering, University of Wollongong, Australia.

3. M. Negnevitsky, Centre for Renewable Energy and Power Systems, University of Tasmania 